\title{
Bifid Median Nerve in the Bulgarian Population: An Anatomical and Clinical Study
}

\author{
Georgi P. Georgiev, ${ }^{1,2}$ Svetoslav A. Slavchev, ${ }^{1}$ Iva N. Dimitrova, ${ }^{3}$ and Boycho Landzhov ${ }^{2}$ \\ ${ }^{1}$ University Hospital of Orthopaedics "Prof. B. Boychev", Medical University, 1614 Sofia, Bulgaria \\ ${ }^{2}$ Department of Anatomy, Histology and Embryology, Medical University, 1431 Sofia, Bulgaria \\ ${ }^{3}$ Department of Cardiology, University Hospital "St. Ekaterina”, Medical University, 1431 Sofia, Bulgaria
}

Correspondence should be addressed to Georgi P. Georgiev; georgievgp@yahoo.com

Received 5 August 2015; Revised 27 October 2015; Accepted 29 October 2015

Academic Editor: Li-Hsien Lin

Copyright (C) 2015 Georgi P. Georgiev et al. This is an open access article distributed under the Creative Commons Attribution License, which permits unrestricted use, distribution, and reproduction in any medium, provided the original work is properly cited.

\begin{abstract}
High division of the median nerve proximal to the carpal tunnel, also known as a bifid median nerve, is a rare anatomical variant with an incidence between 1 and 3\%. In order to study the incidence of this anatomical variation in the Bulgarian population, we examined the upper limbs of 51 formol-carbol fixed human cadavers and also 154 upper limbs undergoing carpal tunnel decompression. We detected one case of bifid median nerve during anatomical dissections and two cases in patients with carpal tunnel syndrome. In one of the clinical cases, the anatomical variation was detected preoperatively by MRI. We discuss different variations of this nerve and emphasize their potential clinical implications.
\end{abstract}

\section{Introduction}

Although well known, the bifid median nerve is a rare entity. It has been detected on anatomical specimens, on imaging studies, or during surgical interventions. There are also reports of a split median nerve accompanied by a persistent median artery or abnormal muscles [1-4]. The bifid median nerve may be associated with carpal tunnel syndrome because of its relatively higher cross-sectional area compared with a nonbifid median nerve [4-6].

Herein, we present the incidence of a bifid median nerve in the Bulgarian population and also emphasize its potential clinical importance.

\section{Materials and Methods}

Over a period of 2 years, 51 formol-carbol fixed human cadavers (30 male, 21 female) were studied to investigate the prevalence of the bifid median nerve. They were all from the autopsy material available at the Department of Anatomy, Histology, and Embryology at the Medical University of Sofia. No medical or surgical history of the cadavers was available.
Over a period of 5 years, 154 upper limbs (78 left upper limbs, 76 right upper limbs) were investigated for the presence of bifid median nerve variation during open carpal tunnel release. The age of the patients was between 39 and 82 years.

The medicolegal office and local Ethic Committee approved this study.

\section{Results}

3.1. Cadaver Dissection. Only in one case, in the right upper extremity of a 69-year-old female cadaver, a bifid median nerve was detected (Figure 1). There was no abnormality in the formation and course of the median nerve till the bifurcation $38 \mathrm{~mm}$ before entering the carpal tunnel. The radial part of the median nerve innervated the thumb and the radial side of the index finger as well as the thenar muscles. The ulnar part of the nerve ensured the sensory innervation of the ulnar side of the index finger, the third finger, and the radial side of the fourth finger. The median artery was presented as a long but slender vessel which originated from the common interosseous artery, descended between the two 


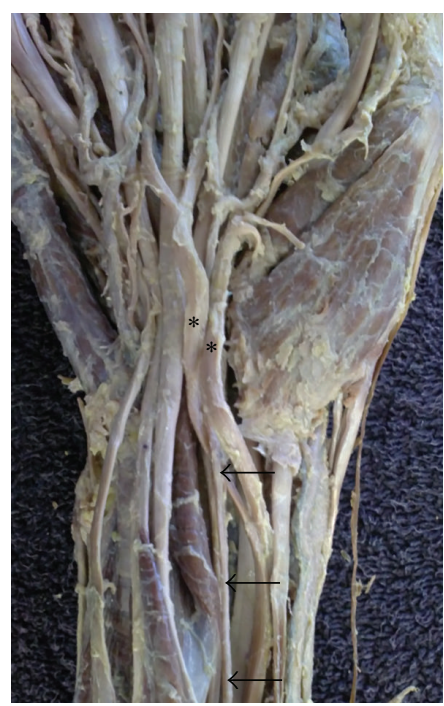

FIGURE 1: Photograph of the wrist and hand of a 69-year-old female cadaver: bifid median nerve (asterisks), median artery (arrows).

parts of the median nerve, passed through the carpal tunnel, and divided into two common digital arteries supplying the thumb and the second finger.

3.2. Surgical Interventions. In 154 upper limbs from patients undergoing carpal tunnel decompression in our hospital, we observed two cases of a bifid median nerve. Both patients, a 39-year-old man and a 65-year-old woman, had no other concurrent diseases and had undergone open surgical carpal tunnel decompression in other hospitals six and seven months of the left nondominant hand and the right dominant hand, respectively. The clinical diagnosis of carpal tunnel syndrome in both patients had been based on a history of nocturnal pain, a sensory deficit in the region of the median nerve distribution, activity-related pain, and loss of handgrip strength together with a positive Phalen's test and a positive Tinel's sign as noted in their discharge summaries. After the first procedure, there had been no improvement of symptoms. During this period, they had had no other orthopaedic evaluation. New electromyographic analysis had presented persistent compression of the median nerve within the carpal tunnel. In the first patient, the MRI obtained before the second surgery revealed a bifid median nerve and incomplete release of the transverse carpal ligament (Figure 2(a)). During the second-look surgery, complete transection of the transverse carpal ligament and neurolysis of both branches of the median nerve were performed. Each branch of the median nerve was supplied by a persistent median artery (Figure 2(b)) and that was also observed in the second patient (Figure 3). In both patients, there was no need of epineurotomy due to the clearly visible fascicles of both parts of the nerve. No aberrant nerve branches and no signs of iatrogenic injury of the median nerve during the carpal tunnel decompression in either patient were detected.

In both, wound healing was uneventful with a volar splint in moderate extension of the wrist for two weeks followed by return to full function as tolerated in each case. Symptoms resolved slowly over the following six months.

\section{Discussion}

The carpal tunnel syndrome in most cases is idiopathic. However, in some cases, a certain etiology can be detected [4]. The symptoms can be provoked by ganglia, neoplastic masses, vascular abnormalities, ligamentous attachments, different anomalous muscles, systemic diseases, obesity, and also a high division of the median nerve [4-9].

The presence of a bifid median nerve varies between 1 and $3 \%$ [10]. In our study, the presence of a bifid median nerve in cadaver dissections is $1.02 \%$ and $3.08 \%$ during surgical procedures, with an overall incidence of $2.05 \%$ in Bulgarians.

Based on a study of 246 hand specimens, Lanz (1977) classified the median nerve variations into 4 groups: (I) variation in the course of the thenar branch, (II) accessory branches at the distal portion of the carpal tunnel, (III) high divisions of the median nerve, and (IV) accessory branches proximal to the carpal tunnel [11]. According to Lanz (1977), the two parts of the nerve are usually equal in size, as in our cases. However, other authors reported a predominance of the radial or ulnar part of the nerve $[2,12]$. Król et al. (2005) stated that the median nerve usually splits in the distal third of the forearm [10].

Although a bifid median nerve is a rare condition, when present, it is usually associated with persistent median vessels [11]. Knowledge of this fact could help orthopedists avoid damage of the coexisting persistent median artery [13].

The presence of a persistent median artery or a bifid median nerve is considered a predisposing factor for the development of carpal tunnel syndrome due to reducing the cross-sectional area of the carpal tunnel $[4-6,9]$. In contrast, Pierre-Jerome et al. (2010) considered that there are no reliable data that the bifid median nerve is the primary cause of carpal tunnel syndrome [14]. Granec et al. (2012) considered that the bifid median nerve could complicate carpal tunnel syndrome symptoms, but there is no evidence that this rare anatomical variation could cause it [13].

The bifid median nerve is also associated with aberrant nerve branches [15]. This should be well known by orthopaedists during carpal tunnel release in order to avoid injury of these branches. Different variant muscles might also be discovered between the two parts of the bifid median nerve. Embryologically, the presence of the anomalous muscle is thought to be the cause of bifidity $[1,16]$.

The two variant parts of the median nerve could pass through separate carpal tunnels. This is clinically important because both tunnels should be released during open or endoscopic surgical treatment of carpal tunnel syndrome [2]. In these cases, epineurectomy in addition to simple decompression of each nerve branch should be performed [13].

Imaging techniques such as ultrasonography and MRI could help in the evaluation and diagnosis of carpal tunnel syndrome and provide useful information about anatomical variations, for example, a bifid median nerve [5]. According to Propeck et al. (2000), these imaging modalities could 


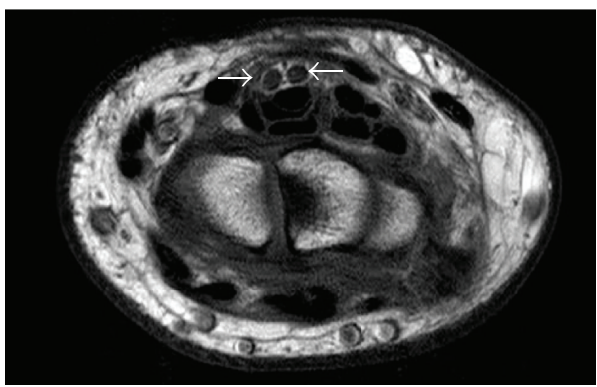

(a)

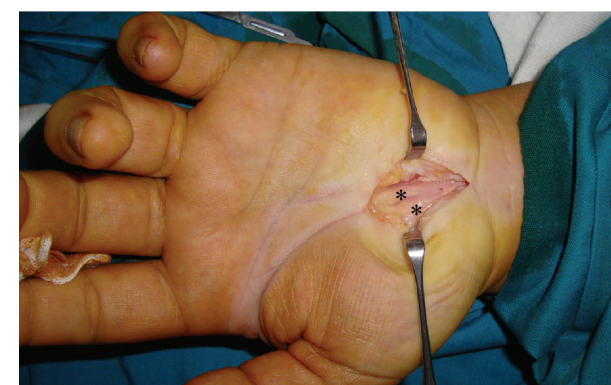

(b)

FIGURE 2: (a) Magnetic resonance image showing the bifid median nerve (arrows) and the remaining portion of the transverse carpal ligament, (b) intraoperative photograph presenting the bifid median nerve (asterisks).

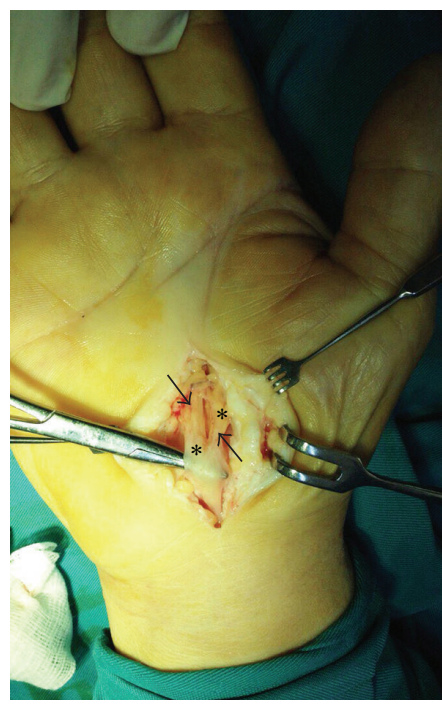

FIGURE 3: Intraoperative photograph presenting the bifid median nerve (asterisks) and median artery (arrows).

clearly present preoperatively the presence of a bifid median nerve. However, they state that the sonographic size criteria for diagnosing carpal tunnel syndrome in nonbifid median nerves may not be accurate for evaluating bifid median nerves [5].

\section{Conclusion}

Although rare, a bifid median nerve should be kept in mind in cases with unilateral or severe carpal tunnel syndrome, failed carpal tunnel release, and early postoperative recurrence. There is no clinical or electrophysiological examination that could reveal bifid median nerve in the carpal tunnel. Therefore, we recommend that an MRI and/or ultrasonography of the carpal tunnel be performed especially in such cases. Certainly, the importance of meticulous surgical technique cannot be overemphasized.

\section{Conflict of Interests}

The authors stated that there is no conflict of interests.

\section{References}

[1] D. P. G. Jones, "Bilateral palmaris profundus in association with bifid median nerve as a cause of failed carpal tunnel release," The Journal of Hand Surgery, vol. 31, no. 5, pp. 741-743, 2006.

[2] M. M. Al-Qattan, K. Al-Zahrani, and M. Al-Omawi, "The bifid median nerve re-visited," The Journal of Hand Surgery: European Volume, vol. 34, no. 2, pp. 212-214, 2009.

[3] A. Hizay and L. Sarikcioglu, "A unique case with splitting of the median nerve by the ulnar artery," Romanian Journal of Morphology and Embryology, vol. 52, no. 2, pp. 723-724, 2011.

[4] A. E. Bagatur, M. Yalcinkaya, and A. O. Atca, "Bifid median nerve causing carpal tunnel syndrome. MRI and surgical correlation," Orthopedics, vol. 36, no. 4, pp. e451-e456, 2013.

[5] T. Propeck, T. J. Quinn, J. A. Jacobson, A. F. G. Paulino, G. Habra, and V. B. Darian, "Sonography and MR imaging of bifid median nerve with anatomic and histologic correlation," American Journal of Roentgenology, vol. 175, no. 6, pp. 1721-1725, 2000.

[6] I. K. Bayrak, A. O. Bayrak, M. Kale, H. Turker, and B. Diren, "Bifid median nerve in patients with carpal tunnel syndrome," Journal of Ultrasound in Medicine, vol. 27, no. 8, pp. 1129-1136, 2008.

[7] T. D. Santoro, H. S. Matloub, and A. K. Gosain, "Ulnar nerve compression by an anomalous muscle following carpal tunnel release: a case report," The Journal of Hand Surgery, vol. 25, no. 4, pp. 740-744, 2000.

[8] R. Luchetti and P. Amadio, "Ethiopathogenesis," in Carpal Tunnel Syndrome, Springer, Berlin, Germany, 6th edition, 2007.

[9] G. P. Georgiev, L. Jelev, and W. A. Ovtscharoff, "Unusual combination of muscular and arterial variations in the upper extremity: a case report of a variant palmaris longus and an additional tendinous portion of the flexor carpi ulnaris together with a persistent median artery," Anatomy, vol. 3, pp. 58-61, 2009.

[10] A. Król, A. Palczak, and K. S. Jedrzejewski, "Split median nerve. A report of two cases," Folia Morphologica, vol. 64, no. 4, pp. 341-344, 2005.

[11] U. Lanz, "Anatomical variations of the median nerve in the carpal tunnel," The Journal of Hand Surgery, vol. 2, no. 1, pp. 4453, 1977.

[12] I. Kessler, "Unusual distribution of the median nerve at the wrist. A case report," Clinical Orthopaedics and Related Research, vol. 67, pp. 124-126, 1969. 
[13] D. Granec, G. Bićanić, I. Borić, and D. Delimar, "Bifid median nerve in a patient with carpal tunnel syndrome-correlation of clinical, diagnostic and intraoperative findings: case report and review of the literature," Acta Clinica Croatica, vol. 51, no. 4, pp. 667-671, 2012.

[14] C. Pierre-Jerome, R. D. Smitson Jr., R. K. Shah, V. Moncayo, M. Abdelnoor, and M. R. Terk, "MRI of the median nerve and median artery in the carpal tunnel: prevalence of their anatomical variations and clinical significance," Surgical and Radiologic Anatomy, vol. 32, no. 3, pp. 315-322, 2010.

[15] N. Z. Winkelman, "Aberrant sensory branch of the median nerve to the third web space-case report," Journal of Hand Surgery, vol. 5, no. 6, pp. 566-567, 1980.

[16] S. Fernandez-Garcia, J. Pi-Folguera, and F. Estallo-Matino, "Bifid median nerve compression due to a musculotendinous anomaly of FDS to the middle finger," The Journal of Hand Surgery, vol. 19, no. 5, pp. 616-617, 1994. 

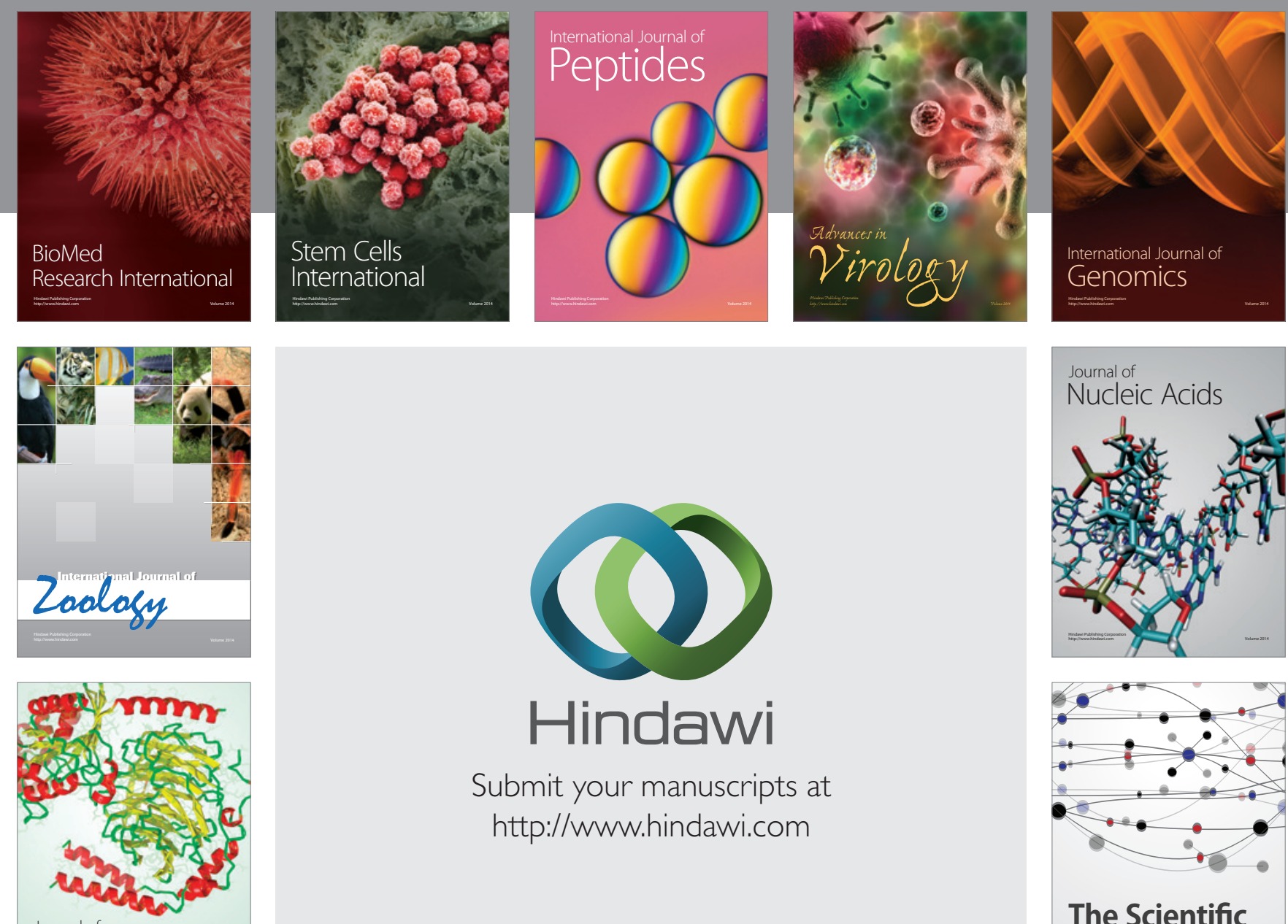

Submit your manuscripts at

http://www.hindawi.com

Journal of
Signal Transduction
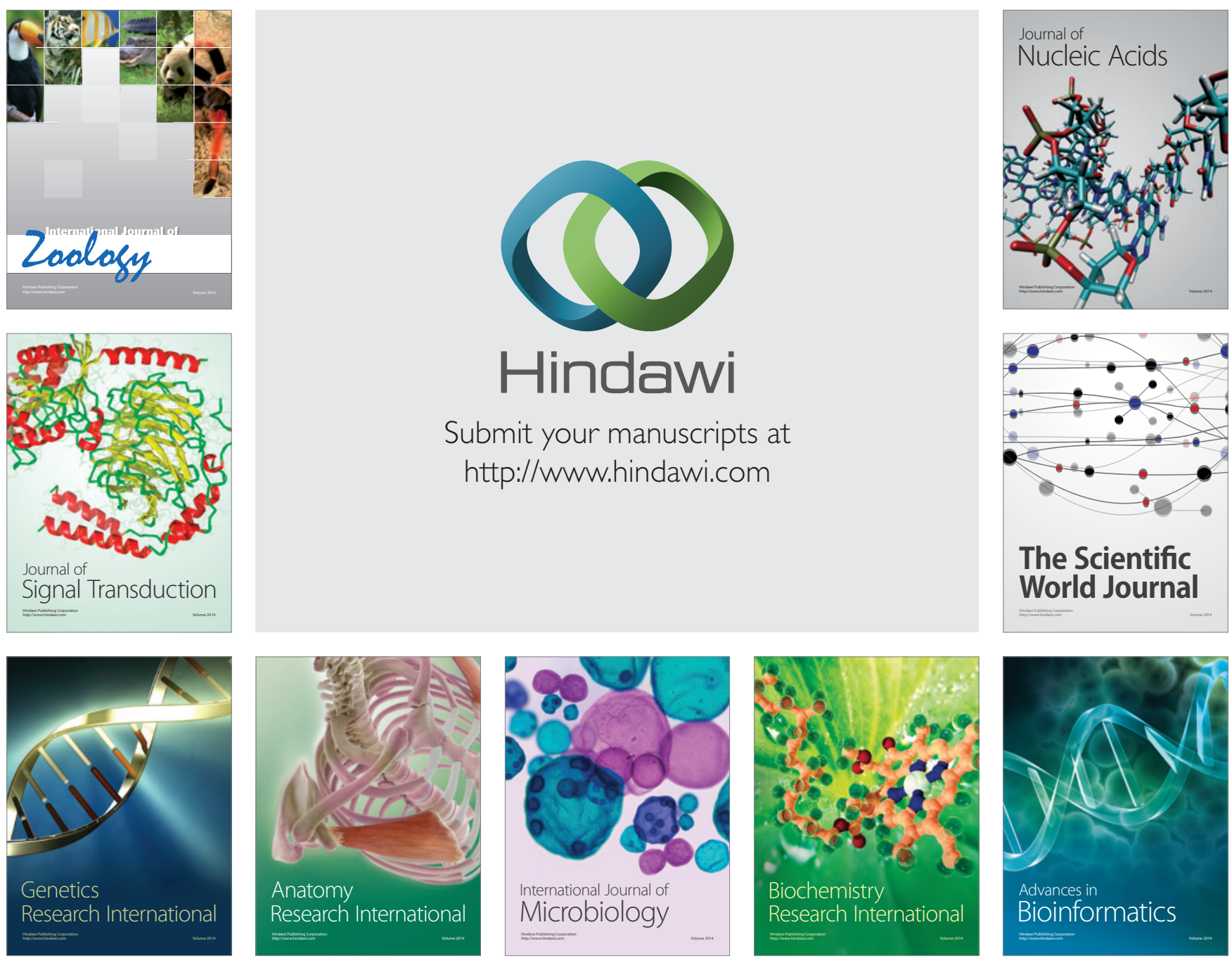

The Scientific World Journal
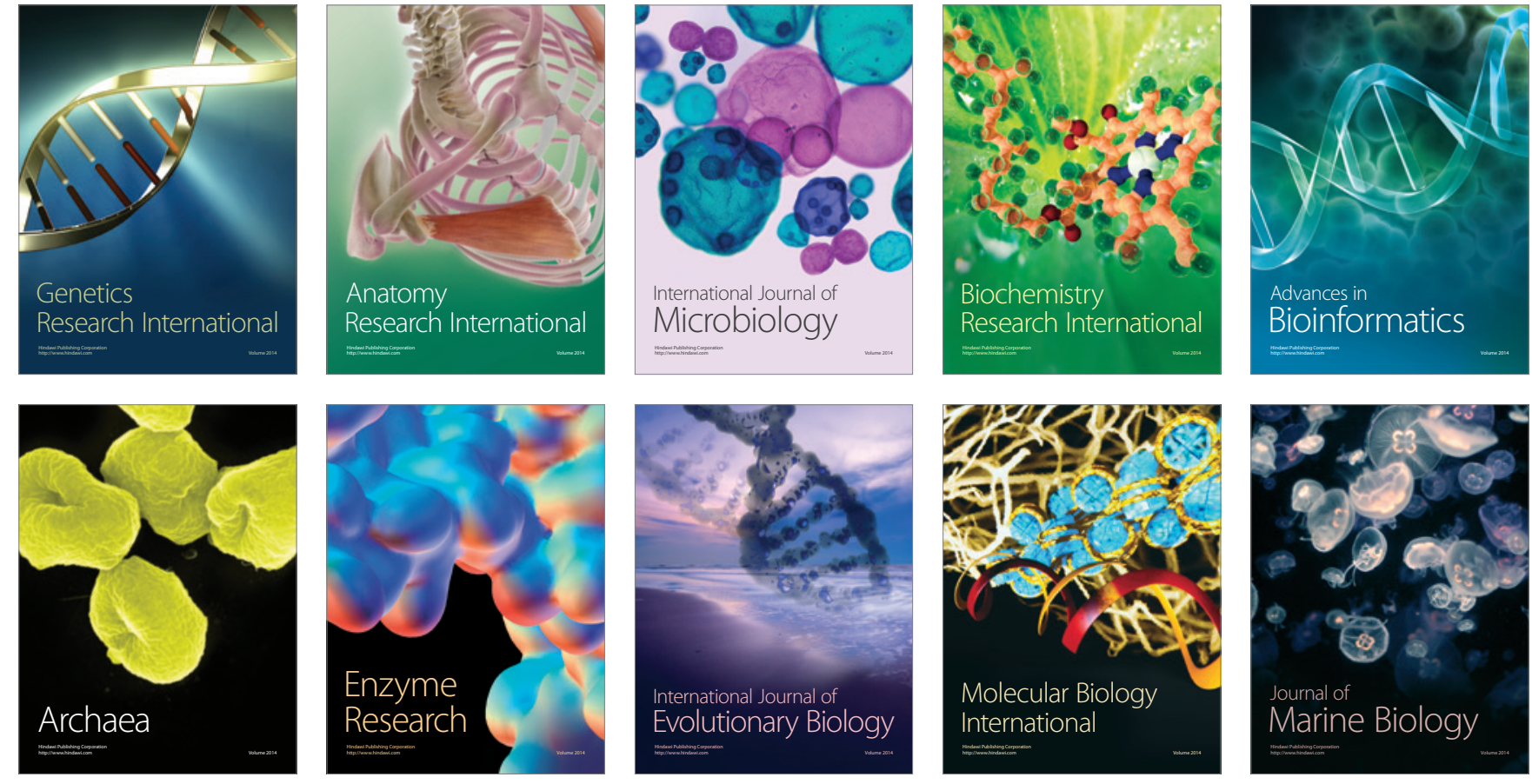